\title{
The Study of RSSI in Wireless Sensor Networks
}

\author{
Jungang Zheng*, Yue Liu, Xufeng Fan and Feng Li \\ Shenyang Jian zhu University, china \\ *Corresponding author
}

\begin{abstract}
Localization estimation of sensor node is a key component in many sensor networks' applications. Correct localization is required for WSN applications, but it is often expensive to include GPS adapters in each sensor node. The RSSI ranged-based localization algorithm is a simple and cost effective localization technology that relies on measuring the Receive Signal Strength Indicator(RSSI) for distance estimation. In this paper, we present experimental results that are carried out to analyze the RSSI model parameter. The least-square linear has been to determine the RSSI model parameter. The results of this study reveal the feasibility and accuracy of RSSI measurement.
\end{abstract}

Keyword-wireless sensor network; RSSI model; distance estimation; node

\section{INTRODUCTION}

Wireless sensor network is composed of thousands of lowcost sensor nodes connected to each other by wireless links. Due to the powerful functions and low energy costs, Wireless sensor networks have been applied in many promising applications such as health surveillance, battle field surveillance, target tracking, environmental monitoring, and so on [1-3].In this application, localization of events is necessary because without the physical localization of events, information related to the events may not be useful. The easiest way to determine node localization is by using global positioning system(GPS), but providing each node with localization hardware GPS receiver is unrealistic, because of the weakness of high cost and energy consuming. Localization problem presents a significant challenge due to limited resources(power and computation capability) of sensor nodes. Hence, localization estimation is a significant technical challenge for the researchers. Localization is one of the key technologies in wireless sensor networks. Interest in wireless sensor networks localization has increased in recent years and many algorithms are developed on this topic. The localization algorithm of the sensor nodes are commonly divided into two categories: range-based algorithm and range-free algorithm[4].The classification is based on whether it is required to measure the actual distances between the nodes or the distance is approximated based on the connectivity information. Range based localization utilizes distance estimation angle information or received signal strength obtained by measuring techniques. Range based algorithm has higher localization accuracy compared with the range-free algorithm. Some well-known range-based algorithms are: RSSI algorithm(received signal strength indicator, TOA algorithm(time of arrival) , AOA algorithm(angle of arrival),TDOA algorithm(time difference on arrival). Rangefree algorithm do not require distance or orientation information between nodes for localization. Range-free algorithm provide cost-effective localization, but their results are less precise than range-based algorithm. Some typical range-free localization algorithm are: APIT algorithm(approximate point-in triangle test), centroid, amorphous, DV-Hop(distance vector-hop)[5].RSSI-based localization algorithm is range-based technology that utilizes the build in RSSI circuitry inside the sensor' transceivers chipsets. RSSI based measurement usually necessitates light computations that do not burden the processing power of the sensor nodes. Hence, it is an economical and cost effective localization technology that gaining an increasing acceptance as a handy technique to solve the localization problem[6-7].In this paper, we focus on the RSSI measurement model. Using least-square linear to find the optimal parameter by the data collecting.

\section{RSSI MODEL AND ERROR ANALYSIS}

The most common range-based technology is based on RSSI measurements. The main idea is to estimate the distance of a transmitter to a receiver using the power of the received signal, knowledge of the transmitted power and the path loss model. At present, the loss model includes Free Space Propagadtion Model, Two-Ray Ground Reflection Model, Log-Distance Path Loss Model, Log-Normal Shadowing Model, and Hadta Model, etc[8].In this paper, we use the LogNormal Shadowing Model. Which is shown in equation(1).

$$
P L(d)=P L\left(d_{0}\right)-10 n \log _{10}\left(\frac{d}{d_{0}}\right)+X_{\sigma}
$$

where PL(d) is the received signal power loss(expressed in $\mathrm{dBm})$ and $\mathrm{d}$ is the distance between a transmitter and receiver. $d_{0}$ is the reference distance,typically $d_{0}=1 \mathrm{~m}$. $\operatorname{PL}\left(\mathrm{d}_{0}\right)$ is the path loss(expressed in $\left.\mathrm{dBm}\right)$ at the reference distance. $\mathrm{n}$ is the path loss exponent, typically $\mathrm{n}=2 \sim 6 . \mathrm{X}_{\sigma}$ is a zero mean Gaussian random variable that reflects the random variation in the path loss due to multipath and shadow fading, namely $X_{\sigma} \sim\left[0, \sigma^{2}\right]$.

In practical application, equation(1) is simplified. The simplified model is shown in (2)[9]

$$
\mathrm{RSSI}=\mathrm{A}-10 \log _{10}(\mathrm{~d})+\mathrm{X}_{\sigma}
$$

where A denotes the received signal strength on reference range, which is $1 \mathrm{~m} . \mathrm{RSSI}=\mathrm{PL}(\mathrm{d})$, which is the actual received signal power at distance $\mathrm{d}, \mathrm{d}$ denotes the transmitter-toreceiver distance.

The simplified model(2) applied in widely conditions, and 
it is utilized as a preferred model in our experiments.

In practical application, the signal strength is affected by three phenomena: path-loss, fading and shadowing[10].Pathloss is the reduction in power density of an electromagnetic wave as it propagates through space. This attenuation is represented by the path-loss exponent, whose value is generally in the range from 2 to 6.Fading is deviation of the attenuation that a signal experience. Shadowing is the loss of signal due to obstacle between a transmitter and receiver. Which have an effect on the model parameter $n$ and A.

In RSSI model, $\mathrm{n}$ and $\mathrm{A}$ can be changed because of such situations as the environment, experimental equipment, etc. Through fitting models based on experimental data, we will explore the error generated by the model in the different ranges of different distance[11].A linear least square approximation has been used to implement the model parameter $\mathrm{n}$ and A. According to the RSSI at different distance the value of parameter $\mathrm{n}$ and $\mathrm{A}$ can be determined.

\section{EXPERIMENT AND SIMULATION}

A key step in RSSI research is the data collection phase, we measure the data with Micaz node, which communicate by chipcon CC2420.CC2420 has powerful communication function. In our experimental rectangular area, nine anchor nodes have been placed, unknown mobile node moves in this rectangular area. Unknown mobile node receives the signal RSSI value in each localization. The nine anchor nodes location are shown in Table I, the unknown mobile node location is shown in Table II,the unknown node receives signal RSSI value is shown in Table III, which really coordinate is $(3.42,2.27)$.

TABLE I. BEACON NODE LOCATION

\begin{tabular}{cccccccccc}
\hline $\mathrm{x}$ & 5.1 & 3.1 & 1.1 & 5.1 & 3.1 & 1.1 & 5.1 & 3.1 & 1.1 \\
\hline $\mathrm{y}$ & 1.6 & 1.6 & 1.6 & 4.1 & 4.1 & 4.1 & 6.6 & 6.6 & 6.6 \\
\hline
\end{tabular}

TABLE II. MOBILE NODE LOCATION

$\begin{array}{lllllllllll}\mathrm{x} & 3.42 & 3.28 & 3.12 & 2.99 & 2.85 & 2.73 & 2.63 & 2.54 & 2.42 & 2.35\end{array}$

$\begin{array}{lllllllllll}\text { y } & 2.27 & 2.43 & 2.57 & 2.63 & 2.89 & 3.04 & 3.2 & 3.4 & 3.57 & 3.76\end{array}$

\section{TABLE III. MEASUREMENT OF BEACON NODE}

$\begin{array}{lrlrrrrrrr}\text { Anchor node } & 1 & 2 & 3 & 4 & 5 & 6 & 7 & 8 & 9 \\ \text { RSSI } & -55.84 & -37.74 & -58.59 & -56.36 & -50.7 & -56.14 & -66.56 & -64.24 & -61.64\end{array}$

The distribution of anchor nodes and unknown mobile node is shown in Figure I.

From Figure I We can see the nine anchor nodes are placed in fixed position, a mobile node(unknown node) moves in a certain position. The anchor nodes and the unknown node have the actual location information in our experiment, which can make us to have an accurate comparison. The unknown node receives the signal RSSI value at different location.

The RSSI-to-relative distance reference curve in Figure II is obtained via a least-square linear fitting from some RSSI measured value.Nine anchor nodes receive the signal RSSI value from one location of the mobile node. From Table I and Table II, the distance between nine anchor nodes and the mobile node can be calculated by Euclidean distance. The model parameter can be achieved by this method.

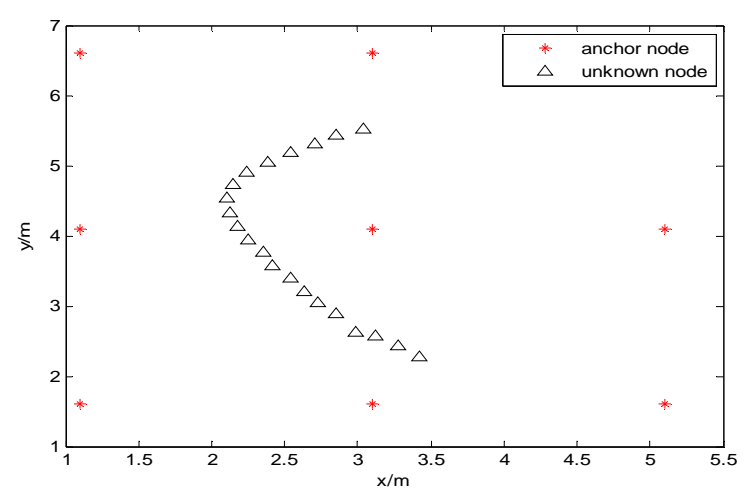

FIGURE I. NODE DISTRIBUTION

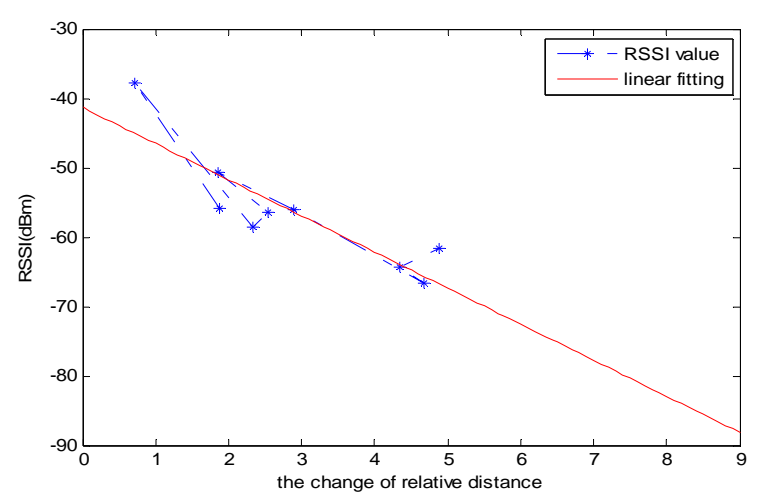

FIGURE II. RSSI-TO-RELATIVE DISTANCE

\section{CONCLUSION}

In this paper, our major objective has been to quantify how good and accurate is the RSSI model in a wireless sensor network to estimate the localization of a target. We study the RSSI model mainly. We study the path loss model. The LogNormal Shadowing Model has been used in this paper. The least-square linear has been to determine the RSSI model parameter. Results show that the RSSI model parameter is suitable. We can get accurate distance by the RSSI model with the parameter. In conclusion the experimental results obtained in this work provide an insight to the feasibility of using RSSI measurements in estimating the location of a target sensor.

\section{ACKNOWLEDGEMENT}

This research was financially supported by the education department of Liaoning province. The grant number is L2015455

\section{REFERENCES}

[1] Cheng L, Wu C, Zhang Y, "A survey of localization in wireless sensor network". International Journal of Distributed Sensor Networks,vol 2012, pp1-12,2012. 
[2] Liao W H, Shih K P, Lee Y C, "A localization protocol with adaptive power control in wireless sensor networks", Computer Communications, vol.31,no.10,pp.2496-2504,2008.

[3] I.F. Akyildiz, W.Su, Y.Sankarasubramaniam, and E.Cayirci, "Wireless sensor network: a survey," Computer Networks(Elesvier), Vol.38, No.4, pp.393-422, 2002.

[4] Wang Y, Fang Z, Chen L, "A New Type of Weighted DV-Hop Algorithm Based on Correction Factor in WSNs" Journal of Communications, vol.9,no.9,pp.699-705,2014.

[5] Kumar S, Lobiyal D K, "Novel DV-Hop localization algorithm for wireless sensor networks" Telecommunication Systems, 2016: 1-16.

[6] Al Alawi R, "RSSI based location estimation in wireless sensors networks"2011 17th IEEE International Conference on Networks. IEEE, pp: 118-122, 2011.

[7] Lim C B, Kang S H, Cho H H, “ An Enhanced Indoor Localization algorithm Based on IEEE 802.11 WLAN using RSSI and multiple parameters"2010 Fifth International Conference on Systems and Networks Communications. IEEE, pp.238-242,2010.

[8] Li J, Zhong X, Lu I T, "Three-dimensional node localization algorithm for WSN based on differential RSS irregular transmission model", Journal of Communications, vol.9,no.5, pp.391-397,2014.

[9] Srbinovska M, Dimcev V, Gavrovski C, et al "Localization Techniques in Wireless Sensor Networks using Measurement of Received Signal Strength Indicator", FACULTY OF ELECTRICAL ENGINEERING UNIVERSITY OF BANJA LUKA, vol.15,no.1,pp.67-71,2011.

[10] Heurtefeux K, Valois F. "Is RSSI a good choice for localization in wireless sensor network", IEEE 26th International Conference on Advanced Information Networking and Applications. IEEE, pp.732-739, 2012.

[11] Cao Z, "Experimental exploration of RSSI model for the vehicle intelligent position system", Journal of Industrial Engineering and Management, vol.8,no.1,pp.51-71,2015. 